\title{
Seroprevalence of Immunoglobulin G Antibody among Contacts of COVID-19 Cases: A Study from India
}

\author{
Om Prakash, MBBS-IAS ${ }^{1}$, Bhavin Solanki, M.D. ${ }^{1}$, Jay Sheth, M.D. ${ }^{2}$, \\ Mehul Acharya, MS-PGDPHM', Mina Kadam, M.D. ${ }^{2}$, Sheetal Vyas, M.D. ${ }^{2}$, \\ Aparajita Shukla, M.D. ${ }^{3}$, Hemant Tiwari, Ph.D. ${ }^{3}$ \\ ${ }^{1}$ Health Department, Ahmedabad Municipal Corporation, Ahmedabad, Gujarat-380001, India. \\ ${ }^{2}$ AMCMET Medical College, Maninagar, Ahmedabad, Gujarat-380008, India. \\ ${ }^{3}$ Smt. NHL Municipal Medical College, Ellisbridge, Ahmedabad, Gujarat-380006, India. \\ Received 23 March 2021 • Revised 26 April 2021 • Accepted 28 April 2021 • Published online 28 June 2021
}

\begin{abstract}
:
Objective: To estimate COVID-19 seropositivity among contacts of cases and to compare the seropositivity among different types of contact for assessing the differential risk \& transmission dynamics.
\end{abstract}

Material and Methods: A large-scale population-based serosurvey was carried out among the general population of Ahmedabad during the second half of October 2020. The contacts of cases were selected based on the population proportion and enrolled as an additional category. The seropositivity among the contacts was estimated using the enzyme-linked immunosorbent assay and compared with different types of contact and available demographic factors.

Results: As of October 2020, the seropositivity against Severe Acute Respiratory Syndrome Coronavirus-2 (SARS-CoV2) among contacts of cases in Ahmedabad was $26.0 \%$ [95\% confidence interval 24.2-28.0]. The seropositivity among family contacts was significantly higher $(28.8 \%)$ compared to other contacts $(24.4 \%)(Z=2.19, p$-value=0.028). This trend was seen across all age groups and both sexes. The seropositivity was higher among females (27.7\%) compared to males $(24.5 \%)$ but the difference was statistically not significant $(Z=1.64, p$-value=0.101). In terms of age groups, the positivity had an increasing trend up to 60 years but declined after that.

Conclusion: A seropositivity of $26.0 \%$ among contacts indicates that a large proportion of contacts demonstrated Immunoglobulin-G antibodies. This highlights asymptomatic transmission and/or low sensitivity of the diagnostic tests. The

Contact: Assoc. Prof. Jay Sheth, M.D.

Department of Community Medicine, AMC MET Medical College,

Maninagar, Ahmedabad, Gujarat-380008, India.

E-mail: jayksheth@yahoo.com

() 2021 JHSMR. Hosting by Prince of Songkla University. All rights reserved.

This is an open access article under the CC BY-NC-ND license

(http://www.jhsmr.org/index.php/jhsmr/about/editorialPolicies\#openAccessPolicy).
J Health Sci Med Res 2022;40(2):147-155 doi: $10.31584 /$ jhsmr.2021823 www.jhsmr.org 
current strategy for contact tracing and testing among contacts is justified based on the significantly higher seropositivity among family contacts.

Keywords: contact of cases, family contact, IgG antibody, SARS-CoV2, serosurveillance

\section{Introduction}

COVID-19, the disease caused by Severe Acute Respiratory Syndrome - Coronavirus 2 (SARS-CoV2), spread across the world during 2020. ${ }^{1,2}$ Being a new virus, the scientific community had little knowledge of its natural history and immune response following a viral infection. ${ }^{3}$ Since the SARS-CoV2 was a novel virus, the presence of antibodies against SARS-CoV2 may be considered as evidence of immunity. This indirect estimation is crucial in assessing the true extent of the SARS-CoV2 spread within a population. ${ }^{4}$ For a newly identified agent, the World Health Organization (WHO) suggests monitoring sero-prevalence. ${ }^{5}$ Since such surveys also uncover asymptomatic, subclinical transmission, it helps in understanding the disease dynamics in a better way and to plan an appropriate public health response. ${ }^{6}$ Through antibody-based serological testing, we can minimize the biases of referral and selective testing affecting laboratory-based surveillance, generate evidence on the role of asymptomatic infection in driving transmission, and estimate the extent of infection. ${ }^{7}$

During the pandemic of COVID-19, multiple serosurveillance studies focusing on the presence of Immunoglobulin-G (IgG) antibody against SARS-CoV2 in general populations and confirmed cases have been carried out across the world. ${ }^{8-10}$ Studies focused on contacts of confirmed cases can add further value to the scientific findings. Seropositivity among contacts, and their comparison with seropositivity among general populations and COVID-19 cases, can give better insight into the risk and dynamics of disease transmission.
Contact with a confirmed case of COVID-19 has been defined by the $\mathrm{WHO}^{11}$, which categorized the contacts into high-risk and low-risk contacts based on the risk of acquiring the infection. High risk contacts include family contacts and others with close contact, which require careful assessment. With the primary objective of estimating seroprevalences, we carried out a sero-survey among contacts of COVID-19 cases. With categorization of our contacts, we could also compare the seropositivity between the two types of contact and to confirm the differential risk \& transmission dynamics.

\section{Material and Methods}

With the objective of estimating the proportion of the population exposed to SARS-CoV2, the Indian Council of Medical Research (ICMR) issued directives for conducting sero-surveys to monitor the pandemic, understand its progression and to take appropriate corrective public health measures. ${ }^{12}$ The Ahmedabad Municipal Corporation (AMC) of the state of Gujarat, India, completed two large scale population-based sero-surveillance surveys for IgG antibodies against SARS-CoV2 during June \& August 2020. A $3^{\text {rd }}$ population-based sero-survey was carried out by AMC during the second half of October 2020, which had the additional categories of Cases, Contacts of cases and Health Care Workers (HCWs) apart from the General population cateogry. Based on our previous 2 studies, we calculated the sample size for the general population category for the 70 Lakh population with $99.0 \%$ confidence and $1.0 \%$ margin of error as 16,600 . Population-based 
stratified sampling was used to decide the required Urban Primary Health Centre (UPHC) target sample size for the general population sample. This was "rounded up" to the next 0 to adjust the sample size of additional categories, which resulted in a general population sample size of 17,000. The sample size for the "contacts of case" category was decided as at least $10.0 \%$ of the general population sample targets and accordingly the Contacts was set at a target sample size of 1,700 . Thus, the contact selection was based on population proportion and unrelated to the COVID-19 cases. This article focuses only on the contacts category of the serosurvey sample.

Family contacts are usually considered as "high risk contacts' as they are more likely to have close contact for longer duration and more likely to have contact without a mask or appropriate Personal Protective Equipment (PPE). Categorizing the contacts into high-risk and lowrisk categories required careful assessment and seemed difficult for field level staff to appropriately categorize the contacts into these 2 categories. For the same reason and for operational feasibility, we categorized the contacts into 'Family contacts' and 'other contacts' for the purposes of our study.

A "Covid Kavach" (Anti SARS-CoV2 IgG Antibody Detection capture Enzyme Linked Immuno Sorbent Assay) kit developed and manufactured by Zydus Diagnostics, validated by National Institute of Virology, Pune, India and approved for use by ICMR was used for the study. The Covid-Kavach has a sensitivity of $92.4 \%$ and a specificity of $97.9 \%$ according to the validation reports. ${ }^{13}$ Thus, with these high levels of sensitivity and specificity, the results received through this testing kit are quite reliable. The manufacturer reported no cross-reactivity with other viruses in the serum from Reverse Transcription Polymerase Chain Reaction test confirmed patients of various other infections. The testing procedures were followed as per the manufacturer's instructions. To reduce the sample rejection rate, Serum Separation Tube - Gel Vacutee was used for the collection of blood samples.

The primary health care network in Ahmedabad city includes 75 UPHCs in 48 wards across 7 zones. Contacts of COVID-19 cases were selected from these 75 UPHCs which are functional units for the COVID-19 management in the field area. Any individual, irrespective of age or sex, who was recorded as a contact of a COVID-19 case (and followed up as part of contact tracing activity) was eligible to be enrolled as a "Contact" in the serosurvey. At the UPHC level, based on informed written consent to participate, contacts were selected from the UPHC field area without any exceptions. An effort was made to cover a wide variety of people of different age groups from both genders and from different localities within the field area of the UPHC. Also, so far as possible, an effort was specifically made to cover at least $10.0 \%$ of contacts as $<18$ years and $>60$ years of age. Before enrollment as a contact, we confirmed that the contacts had not been tested and found to be COVID-19 positive in the past and were otherwise fit for enrollment as a "Contact". Upon selection, these contacts were further recorded as a family contact or other contact, based on the presence or absence of a confirmed case of COVID-19 in his/her family.

The study was carried out after Ethics Committee approval from the AMC MET Medical College. Before enrollment, a written informed consent was received from all the participants. In case of contacts from a minor age group, an assent with informed written consent of their parents/ guardian was obtained for the purpose of the study. Strict confidentiality was ensured at all levels. For the purpose of testing and standardization, only those laboratories with national level accreditation and state of the art facilities and equipment were approved for testing the samples of the study. 
Microsoft Excel and Epi-Info were used for the purpose of data management. In-depth analysis of the collected data and the results of the sample was carried out with focus on the type of contact \& other demographic factors. Simple proportions and appropriate statistical tests were used as required. As an ethical obligation, the results were shared with the concerned local health authorities.

\section{Results}

A total of 2,039 serum samples were collected from the contacts, from which 1 sample was rejected by the laboratory. Results were thus available for 2,038 contacts. From these results $1,489(73.1 \%)$ were negative and 18 $(0.9 \%)$ had indeterminate results. The remaining 531 results were positive for the antibodies against COVID-19 giving an overall positivity of about $26.0 \%$ [95\% Cl 24.2-28.0\%].

Detailed analysis of contacts (Table 1) showed 974 females and 1,064 males. A total of 270 samples were positive among females giving a positivity rate of $27.7 \%$ [95\% Cl 25.0-30.6\%] while 261 samples were positive among males giving a positivity of $24.5 \%$ [95\% Cl 22.0-27.2\%]. This difference between the two sex groups was statistically not significant $(Z=1.64, p$-value $=0.101)$. Seroprevalence was also analyzed for the types of contact (Table 2). The overall seropositivity among family contact was $28.8 \%$ [95\% Cl 25.7-32.1\%] and among other contacts it was $24.4 \%$ [95\% Cl 22.1-26.8\%]. This difference was statistically significant $(Z=2.19, p-v a l u e=0.028)$.

Table 1 Analysis of COVID-19 seropositivity among contacts of cases

\begin{tabular}{|c|c|c|c|c|c|c|c|c|c|c|}
\hline & \multicolumn{3}{|c|}{ Female } & \multicolumn{3}{|c|}{ Male } & \multicolumn{3}{|c|}{ Total } & \multirow[b]{2}{*}{$\begin{array}{l}95 \% \\
\text { confidence } \\
\text { interval }\end{array}$} \\
\hline & 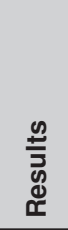 & 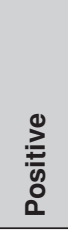 & 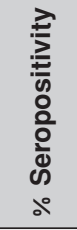 & 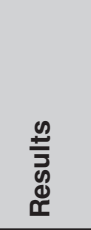 & 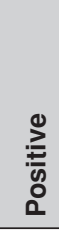 & 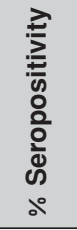 & 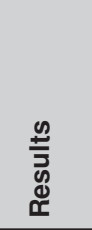 & 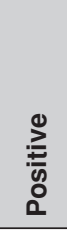 & 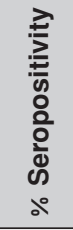 & \\
\hline Total & 974 & 270 & 27.7 & 1,064 & 261 & 24.5 & 2,038 & 531 & 26.0 & $24.2-28.0$ \\
\hline \multicolumn{11}{|l|}{ Type of contact } \\
\hline Family contact & 404 & 117 & 29.0 & 367 & 105 & 28.6 & 771 & 222 & 28.8 & $25.7-32.1$ \\
\hline Other contact & 570 & 153 & 26.8 & 697 & 156 & 22.4 & 1,267 & 309 & 24.4 & $22.1-26.8$ \\
\hline \multicolumn{11}{|l|}{ Age group (years) } \\
\hline $0-9$ & 6 & 1 & 16.7 & 17 & 4 & 23.5 & 23 & 5 & 21.7 & $7.5-43.7$ \\
\hline $10-19$ & 70 & 16 & 22.9 & 95 & 23 & 24.2 & 165 & 39 & 23.6 & $17.4-30.9$ \\
\hline $20-29$ & 253 & 66 & 26.1 & 254 & 48 & 18.9 & 507 & 114 & 22.5 & $19.1-26.3$ \\
\hline $30-39$ & 226 & 61 & 27.0 & 251 & 55 & 21.9 & 477 & 116 & 24.3 & $20.7-28.4$ \\
\hline $40-49$ & 178 & 47 & 26.4 & 192 & 54 & 28.1 & 370 & 101 & 27.3 & 23.0-32.0 \\
\hline $50-59$ & 115 & 41 & 35.6 & 148 & 53 & 35.8 & 263 & 94 & 35.7 & $29.9-41.9$ \\
\hline $60-69$ & 81 & 28 & 34.6 & 75 & 15 & 20.0 & 156 & 43 & 27.6 & 20.7-35.3 \\
\hline $70-79$ & 34 & 8 & 23.5 & 28 & 9 & 32.1 & 62 & 17 & 27.4 & $16.8-40.2$ \\
\hline $80-89$ & 10 & 2 & 20.0 & 4 & 0 & 0.0 & 14 & 2 & 14.3 & $1.8-42.8$ \\
\hline 90-99 & 1 & 0 & 0.0 & - & - & - & 1 & 0 & 0.0 & - \\
\hline
\end{tabular}

Sex difference in seropositivity: $Z=1.64, p-v a l u e=0.101$

Type of contact difference in seropositivity: $Z=2.19, p-$ value $=0.028$ 
Table 2 Age-sex percentage seropositivity according to type of contact

\begin{tabular}{|c|c|c|c|c|c|c|c|c|}
\hline & \multicolumn{4}{|c|}{ Family contact } & \multicolumn{4}{|c|}{ Other contact } \\
\hline & $\begin{array}{l}\frac{0}{\overparen{T}} \\
\frac{\text { हू }}{\Phi}\end{array}$ & $\frac{0}{\frac{0}{\pi}}$ & $\begin{array}{l}\text { 제 } \\
\text { 응 }\end{array}$ & $\begin{array}{l}95 \% \\
\text { confidence } \\
\text { interval }\end{array}$ & $\begin{array}{l}\frac{0}{\pi} \\
\frac{E}{0} \\
\text { एँ }\end{array}$ & $\frac{\frac{0}{\pi}}{\sum}$ & $\begin{array}{l}\bar{\varpi} \\
\stackrel{0}{0}\end{array}$ & $\begin{array}{l}95 \% \\
\text { confidence } \\
\text { interval }\end{array}$ \\
\hline Total & 29.0 & 28.6 & 28.8 & $25.7-32.1$ & 26.8 & 22.4 & 24.4 & $22.1-26.8$ \\
\hline \multicolumn{9}{|l|}{ Age group } \\
\hline $0-9$ & 20.0 & 30.0 & 26.7 & $7.8-55.1$ & 0.0 & 14.3 & 12.5 & $0.3-52.6$ \\
\hline $10-19$ & 25.7 & 28.9 & 27.4 & $17.6-39.1$ & 20.0 & 21.0 & 20.6 & $12.9-30.4$ \\
\hline $20-29$ & 24.4 & 26.2 & 25.3 & $18.8-32.7$ & 26.9 & 15.5 & 21.2 & $17.2-25.8$ \\
\hline $30-39$ & 30.6 & 21.6 & 26.0 & $19.6-33.2$ & 24.8 & 22.1 & 23.4 & $19.0-28.4$ \\
\hline $40-49$ & 27.8 & 29.6 & 28.6 & $21.1-37.0$ & 25.2 & 27.5 & 26.6 & $21.1-32.7$ \\
\hline $50-59$ & 29.1 & 49.0 & 38.7 & $29.4-48.6$ & 41.7 & 28.9 & 33.8 & $26.4-41.7$ \\
\hline $60-69$ & 36.4 & 20.0 & 29.7 & $19.7-41.5$ & 32.4 & 20.0 & 25.6 & $16.6-36.4$ \\
\hline $70-79$ & 40.0 & 28.6 & 34.5 & $17.9-54.3$ & 10.5 & 35.7 & 21.2 & $9.0-38.9$ \\
\hline $80-89$ & 33.3 & 0.0 & 20.0 & $0.5-71.6$ & 14.3 & 0.0 & 11.1 & $0.3-48.2$ \\
\hline $90-99$ & 0.0 & - & 0.0 & - & - & - & - & - \\
\hline
\end{tabular}

Sex difference in seropositivity among Family contacts: $Z=0.11, p$-value $=0.912$

Sex difference in seropositivity among Other contacts: $Z=1.84, p-v a l u e=0.066$

Among family contacts, females had slightly higher positivity $(29.0 \%)$ than males $(28.6 \%)$ and this difference was statistically not significant $(Z=0.11, p$-value $=0.912)$. Among other contacts, females had higher positivity (26.8\%) than males $(22.4 \%)$ and this difference was statistically not significant $(Z=1.84, p-v a l u e=0.066)$. The comparison of seropositivity in both the gender groups, categorized according to type of contact (Figure 1) showed that females had higher seropositivity than males and the other contacts had lower positivity as compared to family contacts.

The age distribution of the contacts of cases typically followed age-heaping bias at 5-year gaps (data not shown, only grouped data shown in data table) as the age of the enrolled individuals were not verified with any official documents and were just recorded as informed. The age of the contacts ranged from 2 years to 93 years with a mode of 30 years, median of 36 years and an average of
$37.93 \pm 15.69$ years. The mean age of the female contacts was $38.43 \pm 15.79$ years, while the mean age of the male contacts was $37.47 \pm 15.59$ years. Considering the seropositive results only, the mean age for the females was $39.67 \pm 15.58$ years and for the males was $39.50 \pm 15.32$ years.

The age group analysis of positivity with type of contacts (Figure 2) showed that the age group with the highest positivity was the 50-59 years group. The seropositivity among contacts had an increasing trend as the age increased up to 60 years, while beyond the age of 60 years it showed declining positivity. When the positivity among contacts was further bifurcated into family contacts and other contacts, the same trend was seen for both types of contacts. The positivity for family contacts was higher than that of other contacts for all age groups. 


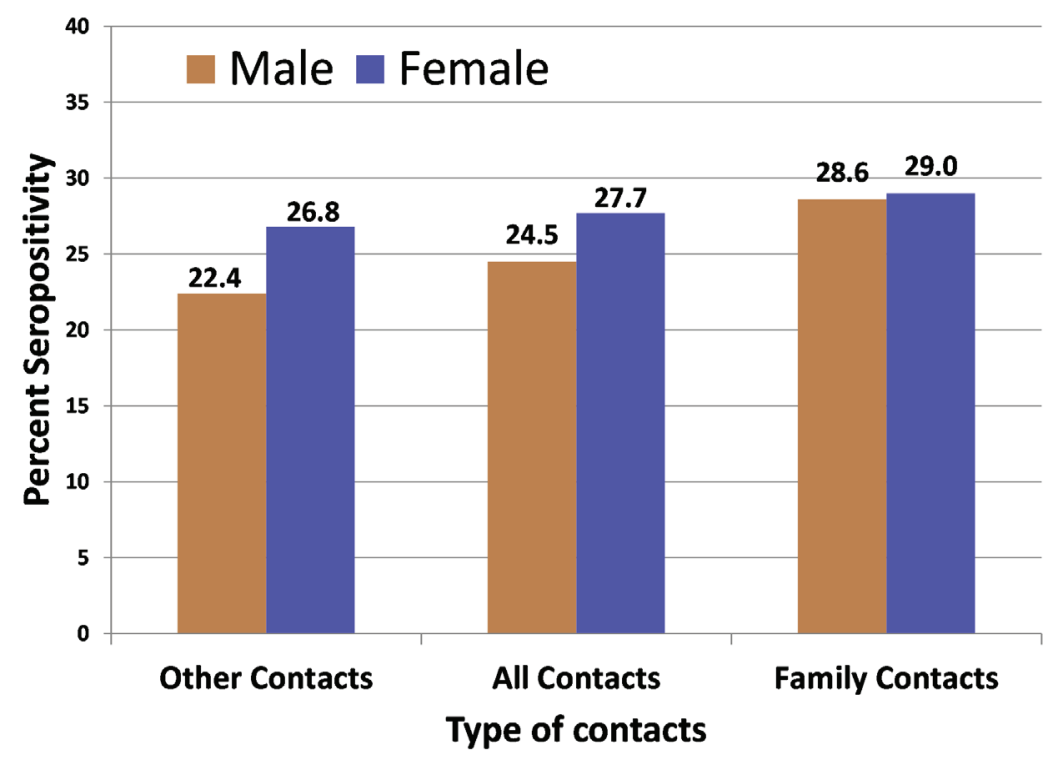

Figure 1 Sex seropositivity according to type of contact

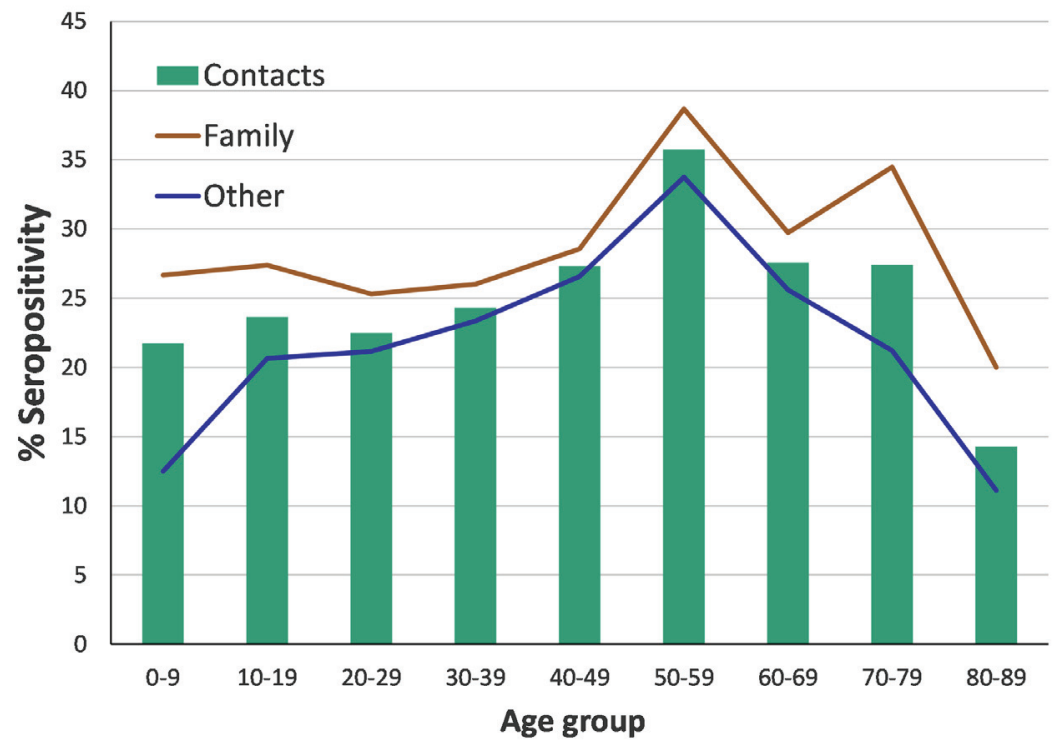

Figure 2 Age group seropositivity according to type of contact 


\section{Discussion}

Although the scientific community is aware of the general immune response after any viral infection, and has some insight into the seropositivity for COVID-19, there is very little information about the immune response among Contacts of COVID-19 cases. ${ }^{14,15}$ The present study on the seropositivity among contacts is among the few serological studies from India, exclusively covering the contacts of COVID-19 cases with a large sample of contacts.

Seropositive contacts are those contacts who demonstrate the presence of IgG antibodies against SARSCoV2 without being tested positive for the COVID-19. This is on account of their contact or exposure with a case. The proportion of seropositive contacts gives an idea of the infected but undetected proportion of contacts. At the same time, these contacts play a crucial role in disease transmission dynamics as they remain as contacts only and they are not identified as clinical or subclinical cases. ${ }^{16}$ In a population-based sample, considering this proportion of seropositive contacts, we can estimate the proportion of the general population who might have already acquired an immune response against SARS-CoV2.

Based on our findings of an average seropositivity of $26.0 \%$ [95\% Cl 24.2-28.0\%] among contacts, it can be said that about a fourth of total contacts had already acquired an immune response without being identified as a confirmed case. Low sensitivity of the diagnostic test along with the presence of a large number of asymptomatic contacts might be the reason for this level of seropositivity. It also uncovers the low sensitivity issue of the diagnostic tests and emphasizes the importance of contact tracing and disease control strategies adopted by the authorities.

Since the contacts in our study were selected based on a population proportion, and the sampling was not related to COVID-19 cases, the proportions of family contacts and other contacts are unrelated. Still, however, the selection of a higher number of other contacts $(1,267)$ than family contact
(771) supports the fact that generally a higher number of casual other contacts are recorded for a confirmed case in contrast to a limited number of family contacts during the process of contact tracing. The positivity among family contacts was significantly higher (28.8\%) compared to other contacts $(24.4 \%)$, a finding which indirectly justifies the risk categorization of family contacts as high-risk contacts and validates the strategy adopted for these high-risk contacts.

Considering the seropositivity among sexes, there was a narrow difference among family contacts as compared to the other contacts where the difference was high but statistically not significant. This suggests that the higher risk among family contacts affects both the gender groups equally. Although the sex difference in positivity was not significant, the consistent finding of higher seropositivity among females as compared to males, irrespective of the type of contact, requires further in-depth analyses to learn the reasons for this trend.

The statistic of mode $<$ median $<$ mean also indicates that the distribution had many young adults as compared to elderly and the mean deviates on the right due to the higher values of a comparatively small number of elderly contacts with age more than double the mean age. Considering the age-group seropositivity (Figure 2) the family contacts had higher seropositivity than the other contacts for all age groups. The age group trend for seropositivity was similar for both types of contacts but the difference was quite large for the child and adolescent groups. This can be explained by the higher susceptibility among children and adolescents upon exposure, particularly as family contacts. These groups might also have lower compliance with preventive measures/strategies, leading to subsequent seroconversion.

For adults the seropositivity increased with increasing age up to 60 years and thereafter the seropositivity reduced. This trend was seen for both types of contacts. The decreasing trend beyond 60 years may have been due to the small sample size in these age groups and 
restricted work-related mobility among them, particularly in lock-down and partial unlocked situations. According to the scientific studies, young adults are more likely to be asymptomatic as compared to the elderly, and even when such contacts are symptomatic, the symptoms are more likely to be mild and for shorter duration among the young adults than the elderly. ${ }^{17-22}$ This difference in symptoms among the contacts may be the reason behind the higher seroconversion among the elderly with the increasing trend in positivity with increasing age. However, in the absence of data on symptoms and their severity and duration, we could only presume but could not confirm the above-mentioned reasons.

The present study gives an estimate of contacts who might have already acquired immunity on account of their contact with a case, without being tested positive. The proportion of seropositivity among contacts justifies the adopted national strategy to test all high-risk contacts and symptomatic low-risk contacts. If contacts are not identified by contact tracing and kept in quarantine (with subsequent testing when indicated, if at all) then the ongoing transmission could be exponential. So [no comma]this study also reaffirms the need for a contact tracing strategy for controlling the inevitable spread of the COVID-19 pandemic. The results have highlighted the scope for further research to generate greater evidence regarding the risk dynamics and COVID-19 transmission.

\section{Conclusion}

Overall seropositivity of $26.0 \%$ among the contacts gives an indirect estimate that about a fourth of the total contacts already had antibodies against SARS-CoV2. This highlights the fact of asymptomatic/subclinical transmission and the low sensitivity of the diagnostic tests applied for testing for COVID-19. This also reaffirms the need for a contact tracing strategy for controlling the inevitable spread of the pandemic. Higher positivity among family contacts justifies the risk categorization and testing strategy adopted for the contacts of the cases. The results have highlighted the scope for further research to generate greater evidence regarding the risk dynamics and COVID-19 transmission.

\section{Acknowledgement}

We are extremely thankful to respected Dr. Rajiv Kumar Gupta, IAS (Additional Chief Secretary, Government of Gujarat) and Mr. Mukesh Kumar, IAS (Municipal Commissioner, Ahmedabad) for their wholehearted support. We acknowledge the full support from the field level health care workers (Corona warriors). All the Zonal Deputy Health Officers, Deputy Health Officer (Epidemic), Assistant Health Officers and Medical officers of the UPHCs extended their full support in conducting the serosurveillance. We are thankful to all the medical and paramedical support staff involved with the study. Finally, we are indebted to all the participants whose willingness and support generated the much-desired data for the study.

\section{Conflict of interest}

We declare no conflicts of interest

\section{References}

1. Munster VJ, Koopmans M, Van Doremalen N, Van Riel D, Emmie de Wit. A Novel Coronavirus Emerging in China A Novel Coronavirus Emerging in China-Key Questions for Impact Assessment. N Engl J Med 2020;38:692-4.

2. Li Q, Guan X, Wu P, Wang X, Zhou L, Tong Y, et al. Early transmission dynamics in Wuhan, China, of novel coronavirusinfected pneumonia. N Engl J Med 2020;382:1199-207.

3. Lipsitch M, Swerdlow DL, Finelli L. Defining the epidemiology of Covid-19 - studies needed. N Engl J Med 2020;382:1194-6.

4. GeurtsvanKessel $\mathrm{CH}$, Okba NMA, Igloi Z, Bogers S, Embregts CWE, Laksono BM, et al. An evaluation of COVID-19 serological assays informs future diagnostics and exposure assessment. Nat Commun 2020;11:3436.

5. World Health Organization. Population-based age-stratified seroepidemiological investigation protocol for COVID-19 virus infection. Geneva: WHO; 2020. 
6. Hanage WP, Qiu X, Kennedy-Shaffer L. Snowball sampling study design for serosurveys in the early COVID-19 pandemic. Dash Havard Edu 2020;1-12.

7. Kumar MS, Bhatnagar T, Manickam P, Kumar VS, Rade K, Shah N, et al. National sero-surveillance to monitor the trend of SARS-CoV-2 infection transmission in India: Protocol for community-based surveillance. Indian J Med Res 2020; $151: 419-23$

8. Xu X, Sun J, Nie S, Li H, Kong Y, Liang M, et al. Seroprevalence of immunoglobulin $\mathrm{M}$ and $\mathrm{G}$ antibodies against SARS-CoV-2 in China. Nat Med 2020;26:1193-5.

9. Murhekar M V, Bhatnagar T, Selvaraju S, Rade K, Saravanakumar V, Wesley Vivian Thangaraj J, et al. Prevalence of SARSCoV-2 infection in India: findings from the national serosurvey, May-June 2020. Indian J Med Res 2020;152:48-60.

10. Hou $\mathrm{H}$, Wang $\mathrm{T}$, Zhang $\mathrm{B}$, Luo $\mathrm{Y}$, Mao L, Wang $\mathrm{F}$, et al Detection of IgM and IgG antibodies in patients with coronavirus disease 2019. Clin TransI Immunology 2020;9:e01136.

11. World Health Organization. Public health surveillance for COVID-19: interim guidance. Geneva: WHO; 2020.

12. Indian Council of Medical Research. Press Release ICMR advises States to conduct sero-survey to measure Coronavirus exposure in the population using IgG ELISA Test. New Delhi: Indian Council of Medical Research; 2020.

13. Sapkal G, Shete-Aich A, Jain R, Yadav PD, Sarkale P, Lakra R, et al. Development of indigenous IgG ELISA for the detection of anti-SARS-CoV-2 IgG. Indian J Med Res 2020;151:444-9.

14. Chirathaworn $C$, Sripramote $M$, Chalongviriyalert $P$, Jirajariyavej S, Kiatpanabhikul P, Saiyarin J, et al. SARS-CoV-2 RNA shedding in recovered COVID-19 cases and the presence of antibodies against SARS-CoV-2 in recovered COVID-19 cases and close contacts, Thailand, April-June 2020. PLoS One 2020;15: e0236905.
15. Martinez-Fierro ML, Ríos-Jasso J, Garza-Veloz I, Reyes-Veyna L, Cerda-Luna RM, Duque-Jara I, et al. The role of close contacts of COVID-19 patients in the SARS-CoV-2 transmission: an emphasis on the percentage of nonevaluated positivity in Mexico. Am J Infect Control 2021;49:15-20.

16. Havers FP, Reed C, Lim T, Montgomery JM, Klena JD, Hall AJ, et al. Seroprevalence of Antibodies to SARS-CoV-2 in 10 Sites in the United States, March 23-May 12, 2020. JAMA Intern Med 2020;180:1776-86.

17. Wang X, Guo X, Xin Q, Pan Y, Hu Y, Li J, et al. Neutralizing Antibodies Responses to SARS-CoV-2 in COVID-19 Inpatients and Convalescent Patients [published online ahead of print, 2020 Jun 4]. Clin Infect Dis 2020. doi: 10.1093/cid/ciaa721.

18. Hu WT, Howell JC, Ozturk T, Benameur K, Bassit LC, Ramonell $R$, et al. Antibody Profiles According to Mild or Severe SARSCoV-2 Infection, Atlanta, Georgia, USA, 2020. Emerg Infect Dis 2020;26:2974-8.

19. Okba NM, Müller MA, Li W, Wang C, GeurtsvanKessel CH, Corman VM, et al. Severe Acute Respiratory Syndrome Coronavirus 2-Specific Antibody Responses in Coronavirus Disease Patients. Emerg Infect Dis 2020;26:1478-88.

20. Kellam P, Barclay W. The dynamics of humoral immune responses following SARS-CoV-2 infection and the potential for reinfection. J Gen Virol 2020;101:791-7.

21. Cervia C, Nilsson J, Zurbuchen Y, Valaperti A, Schreiner J, Wolfensberger $A$, et al. Systemic and mucosal antibody responses specific to SARS-CoV-2 during mild versus severe COVID-19. J Allergy Clin Immunol 2021;147:545-57.e9.

22. Nikolai LA, Meyer CG, Kremsner PG, Velavan TP. Asymptomatic SARS Coronavirus 2 infection: Invisible yet invincible. Int $\mathrm{J}$ Infect Dis 2020;100:112-6. 